\title{
Crystallographic Orientation Analyses of Magnetite Thin Films Using Electron Backscatter Diffraction (EBSD)
}

\author{
A. Koblischka-Veneva ${ }^{1}$, M. R. Koblischka ${ }^{2}$, F. Mücklich ${ }^{1}$, S. Murphy ${ }^{3}$, Y. Zhou ${ }^{3}$, and I. V. Shvets ${ }^{3}$ \\ ${ }^{1}$ Institute of Functional Materials, University of the Saarland, D-66041 Saarbrücken, Germany \\ ${ }^{2}$ Institute of Experimental Physics, University of the Saarland, D-66041 Saarbrücken, Germany \\ ${ }^{3}$ SFI Nanoscience Laboratory, Trinity College, Dublin 2, Ireland
}

\begin{abstract}
The crystallographic orientation of magnetite $\left(\mathrm{Fe}_{3} \mathrm{O}_{4}\right)$ thin films was measured using electron backscatter diffraction (EBSD). Misorientation boundaries appear in maps of angular misorientation data. The distribution of misorientation angles changes after annealing the samples in air at $250^{\circ} \mathrm{C}$. Most small-angle misorientations $\left(<5^{\circ}\right)$ are removed after one minute of annealing, whereas larger misorientations (as high as $60^{\circ}$ ) continue to persist.
\end{abstract}

Index Terms-Crystal growth, electron microscopy, electron backscatter diffraction, magnetite, position measurement.

\section{INTRODUCTION}

$\mathbf{M}$ AGNETITE $\left(\mathrm{Fe}_{3} \mathrm{O}_{4}\right)$ is a half-metallic ferromagnet spinel material which attracts a lot of interest from the spin electronics community. Therefore, the growth mechanism of epitaxial thin films on substrates like $\mathrm{MgO}$ is very important to be understood. Due to the difference in lattice parameters of magnetite and $\mathrm{MgO}$, which is just half of magnetite, antiphase boundaries (APBs) exist in these films, resulting in half-lattice period shifts between some of the nucleation islands [1]-[3]. In recent studies [4]-[6], it was found that upon a short annealing in air, the magnetic properties of magnetite thin films change significantly, i.e., the coercivity reduces and the samples become much easier to be saturated. As a reason for this behavior, the exchange interaction at the APBs is considered [4], [5]. All these findings favour a detailed analysis of the microstructure of the magnetite films to be carried out employing a high spatial resolution. This goal can be achieved using the electron backscatter diffraction (EBSD) technique, which works within a scanning electron microscope, enabling a spatially resolved study of crystallographic orientations whilst recording Kikuchi patterns. With the recent developments concerning the image recording system, a spatial resolution of about $50 \mathrm{~nm}$ can be achieved on oxidic samples [7], [8]. Therefore, we analyze here the crystallographic orientations of magnetite thin films in as-grown condition and after several annealing steps in air in order to find reasons for the changes in the magnetic properties.

\section{EXPERIMENTAL PROCEDURE}

Epitaxial magnetite thin films with a thickness of $100 \mathrm{~nm}$ were grown on $\mathrm{MgO}$ single-crystal substrates cut along the (1 0 0 ) direction within $\pm 0.1^{\circ}$ by oxygen-plasma-assisted molecular beam epitaxy. The magnetite layer was deposited by means of $e$-gun evaporation from $\mathrm{Fe}$ pellets with a purity of $99.995 \%$ in

Digital Object Identifier 10.1109/TMAG.2006.880079 a plasma oxygen environment of $1 \times 10^{-5}$ Torr with a substrate temperature of $250^{\circ} \mathrm{C}$. Growth mode and crystalline quality of the films were monitored in situ by reflection high-energy electron diffraction (RHEED) [4]. The film thickness was controlled by quartz-crystal thickness monitors calibrated using X-ray reflectivity. More details about the samples preparation can be found in [4]-[6]. The samples were subsequently annealed in air at $250^{\circ} \mathrm{C}$; except sample $\mathrm{S} 1$ (as-grown). For the present study the duration for the annealing was varied between 1 (sample S2), 30 (sample S3), and 180 min (sample S4). This temperature is chosen to be low enough to avoid a direct formation of $\gamma-\mathrm{Fe}_{2} \mathrm{O}_{3}$ as indicated by the Verwey transition and the magneto-resistance values measured in [4], [5]. The longer annealed samples S3 and S4 may contain an amount of $\gamma-\mathrm{Fe}_{2} \mathrm{O}_{3}$.

The EBSD system employed here consists of a FEI dual beam workstation (Strata DB 235) equipped with a TSL OIM analysis unit [9]. The Kikuchi patterns are generated at an acceleration voltage of $20 \mathrm{kV}$, and are recorded by means of a DigiView camera system, allowing a maximum recording speed of the order of $0.05 \mathrm{~s} /$ pattern. The time employed in the case of a multiphase scan is much longer, of the order of $0.4 \mathrm{~s} / \mathrm{pat}$ tern, as a higher image quality/confidence index is required. To produce a crystallographic orientation map, the electron beam is scanned over a selected surface area and the resulting Kikuchi patterns are indexed and analysed automatically (i.e., the Kikuchi bands are detected by means of the software). An image quality (IQ) parameter and a confidence index (CI) is recorded for each Kikuchi pattern. The dimensionless IQ parameter is the sum of the detected peaks in the Hough transform employed in the image recording; the CI value yields information about how exact the indexation was carried out. The CI value ranges between 0 and 1 [9]. Based on the analysis of the recorded CI value, a multiphase analysis is realized. A detailed description of the measurement procedure can be found in [10] and [11]. The results of the EBSD measurement are presented in form of maps, the most important thereof are the so-called inverse pole figure (IPF) maps, indicating the crystallographic orientation of each individual point. Automated EBSD scans 

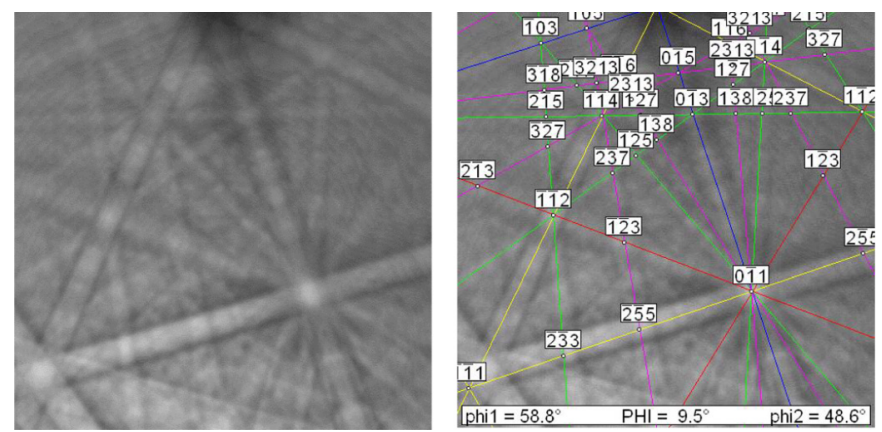

Fig. 1. Kikuchi pattern and the corresponding indexation of magnetite. (Color version available online at http://ieeexplore.ieee.org.)

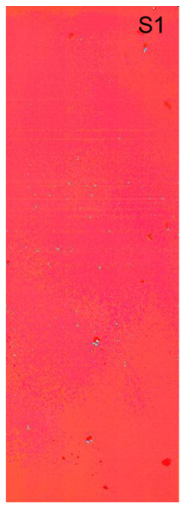

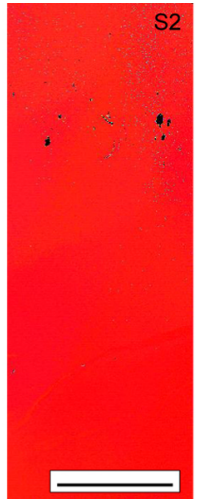

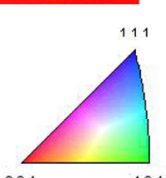

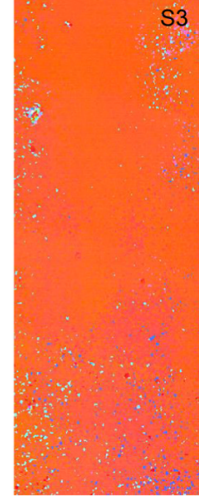
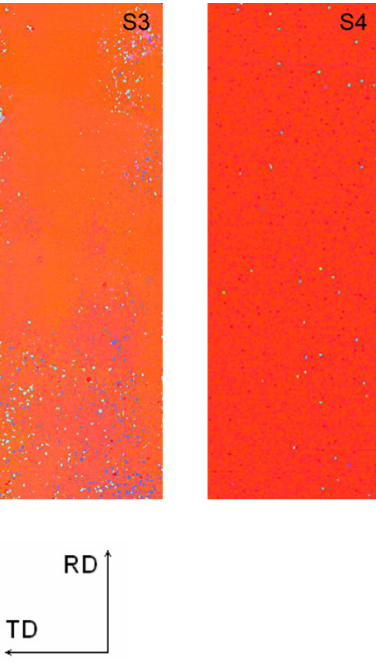

Fig. 2. Inverse pole figure maps in [0 $\left.\begin{array}{lll}0 & 0 & 1\end{array}\right]$ direction (i.e., normal to the sample surface) of the magnetite films S1-S4. The stereographic triangle below gives the color code of the crystal orientation. The maps reveal that the dominant orientation for all samples is [ $\left[\begin{array}{lll}0 & 0 & 1\end{array}\right]$; however, the shade of red is not uniform in all samples. The indicated scale bar is $400-\mu \mathrm{m}$ long. (Color version available online at http://ieeexplore.ieee.org.)

were performed for each sample twice; one with a step size of 1 $\mu \mathrm{m}$ as overview and a detail scan with a step size of $50 \mathrm{~nm}$. The distance between sample and phosphor screen is set at $10 \mathrm{~mm}$.

\section{RESULTS AND DISCUSSION}

Fig. 1 shows an example of measured magnetite Kikuchi patterns and their indexed solution provided by the TSL software. The determined Eulerian angles are $\varphi_{1}=58.8^{\circ}, \Phi=9.5^{\circ}$, and $\varphi_{2}=48.6^{\circ}$. The IQ value achieved is about 300 , which is very high for an oxidic ceramic sample; thus automated multiphase scans are possible to be performed. As compared to the earlier EBSD analysis by Tepper et al. [12], the IQ values recorded here are considerably higher. The Kikuchi patterns clearly reveal the differences between magnetite and maghemite $\left(\gamma-\mathrm{Fe}_{2} \mathrm{O}_{3}\right)$; however, in this paper, we do not discuss the differences between these tow components upon annealing. The determined CI value for the magnetite Kikuchi pattern shown is 1, i.e., a perfect indexation.

Fig. 2, presents the EBSD-determined inverse pole figure (IPF) maps in [l $\left.\begin{array}{lll}0 & 0 & 1\end{array}\right]$ direction. The color indicates the crystallographic orientation according to the stereographic triangle.
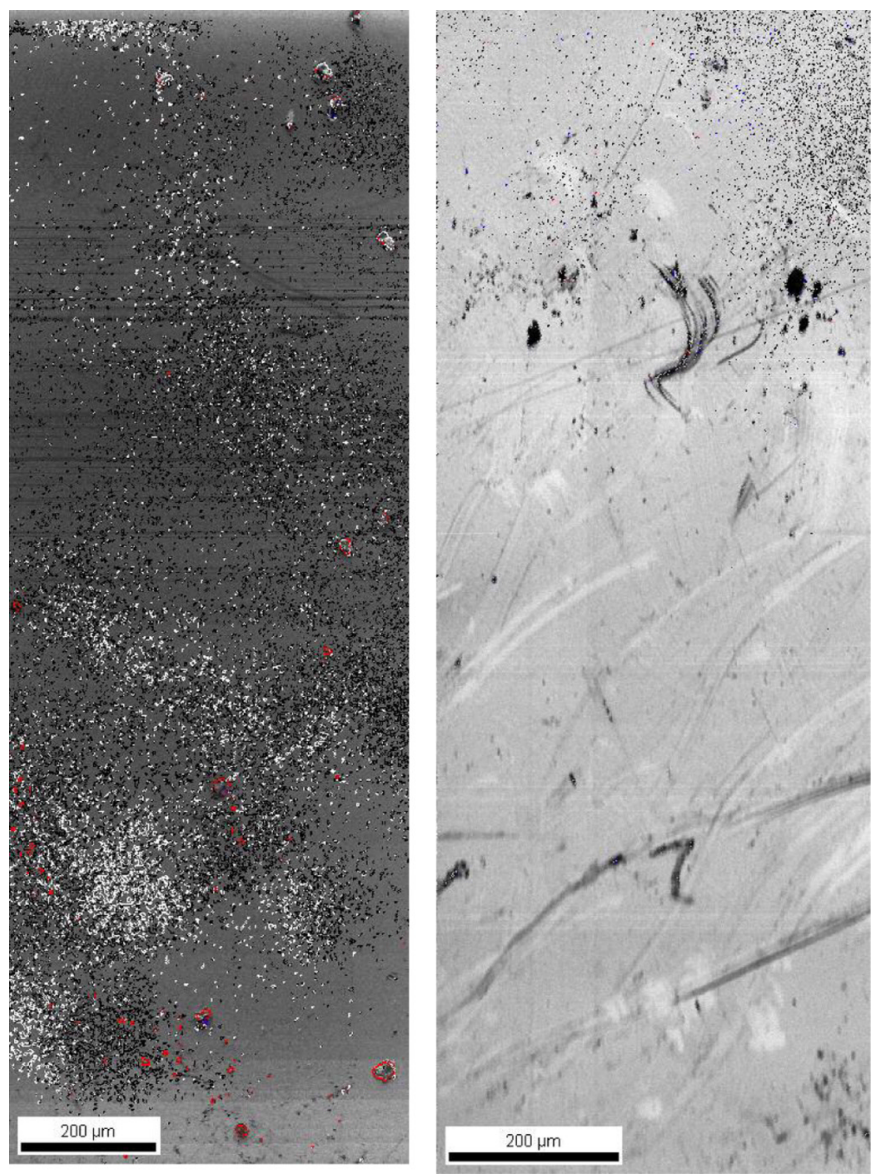

Fig. 3. Image quality (IQ) maps of samples $\mathrm{S} 1$ and $\mathrm{S} 2$, together with the marked misorientation boundaries. Black are boundaries between 2 and $3^{\circ}$, white between 3 and $5^{\circ}$, red between 5 and $15^{\circ}$, and blue between 15 and $30^{\circ}$. The map of sample $\mathrm{S} 2$ shows only some boundaries in range between 2 and $3^{\circ}$. (Color version available online at http://ieeexplore.ieee.org.)

These maps are measured with a step size of $1 \mu \mathrm{m}$. As expected from epitaxial thin films, the selected areas are mainly oriented in $\left[\begin{array}{lll}0 & 0 & 1\end{array}\right]$ direction. However, from these maps we learn that the as-grown sample has not yet developed the optimum orientation, which clearly changes upon annealing (shade of red). The selected area of each sample corresponds to one large grain. Furthermore, there are some characteristic misorientations within the magnetite yielding misorientation angles larger than $60^{\circ}$. These areas have a diameter of about $100 \mathrm{~nm}$, and a typical spacing of $500 \mathrm{~nm}$, which is similar to the distance of antiferromagnetic pinning sites found in the MFM-measurements of [13].

In Fig. 3, we compare the distribution of misorientation angles between samples S1 and S2. Plotted is here the IQ parameter, which gives an indication of the perfection of the crystal lattice in the diffracting volume [9]. This implies that the bright stripes are e.g., flat terraces on the surface, whereas darker areas may indicate dislocations, cracks and voids. The misorientation angle boundaries are indicated in different colors for the different angles $\left(2-3^{\circ}\right.$ black, $3-5^{\circ}$ white, $5-15^{\circ}$ red, and $15-45^{\circ}$ blue). Such a spatially resolved analysis of the grain misorientation is one of the strengths of the EBSD technique. In sample S1, there are a large number of angular misorientations between 2 and $5^{\circ}$, and a small number of high-angle misorientations above 


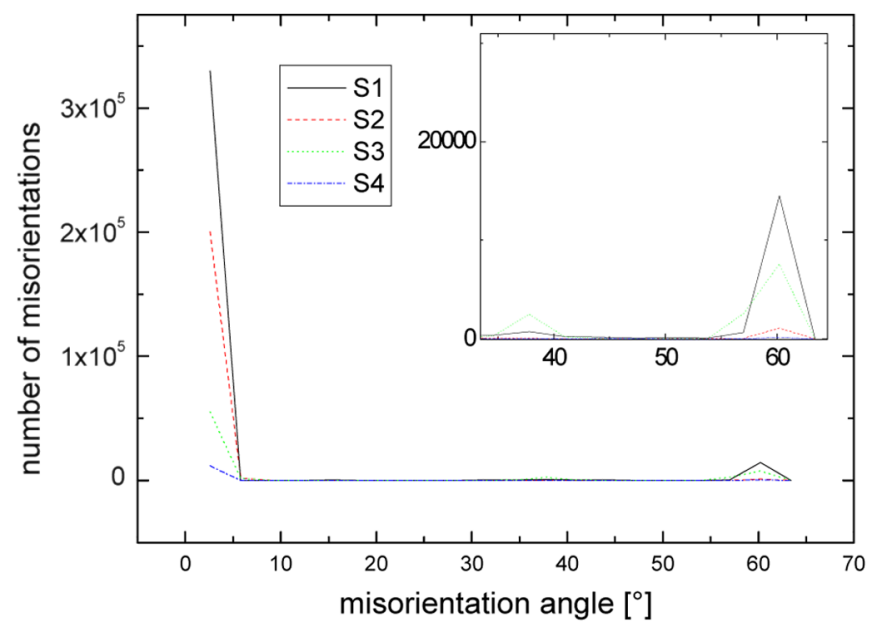

Fig. 4. Misorientation angle graph for all four samples S1-S4 under study, giving the effective number of misorientations as function of the misorientation angle. The inset shows the high-angle part in detail. (Color version available online at http://ieeexplore.ieee.org.)

$5^{\circ}$. The high-angle misorientations are mainly due to small, embedded particles of $\mathrm{MgO}$, which can also be detected by the EBSD system via a two-phase analysis. Upon annealing in air, there is a change in the distribution of misorientation angles: In sample S2, the amount of the small-angle misorientations is considerably reduced. In the selected area, there are only boundaries in the range $2-3^{\circ}$ left, and all the white boundaries $\left(3-5^{\circ}\right)$ are removed. In sample $\mathrm{S} 1$, all these boundaries together span a length of $9.7 \mathrm{~cm}$, whereas in sample S2 only an overall length of $6.5 \mathrm{~mm}$ is measured. In contrast, the number of high-angle misorientations and their cumulative length remain unchanged after annealing.

Fig. 4 summarizes the misorientation analysis by comparing the number of misorientations for each sample; the inset presents the high-angle part in detail. This diagram indicates that a large number of small-angle misorientations (below $5^{\circ}$ ) are present in the films S1 and S2. The annealing in air removes most of these small-angle misorientations, and further, the high-angle misorientations stay mostly unchanged. Sample S3 shows even an increase of the amount of the large-angle misorientations upon annealing, and sample S4 has practically lost all misorientations except at $60^{\circ}$. These high-angle boundaries form finally misoriented small islands (i.e., the light-blue spots in the [0 0 l 1 1] IPF maps of Fig. 2, samples S3 and S4).

\section{CONCLUSION}

The EBSD technique successfully measures the crystallographic orientation of magnetite thin films. A relatively high IQ enables a two-phase scan to be carried out. Our measurements reveal that upon annealing the films in air, the amount of small-angle misorientations reduces considerably; leading to very homogeneous thin films. These changes in the microstructure upon annealing may explain the considerable change in the magnetic properties as found earlier.

\section{ACKNOWLEDGMENT}

This work was supported by DFG-project MU959/12 and the EU-funded project "ASPRINT."

\section{REFERENCES}

[1] S. Celotto, W. Eerenstein, and T. Hibma, "Characterization of antiphase boundaries in epitaxial magnetite films," Eur. J. Phys. B, vol. 36, pp. 271-279, 2003.

[2] D. T. Margulies, F. T. Parker, F. E. Spada, R. S. Goldman, J. Li, R. Sinclair, and A. E. Berkowitz, "Anomalous moment and anisotropy behavior in $\mathrm{Fe}_{3} \mathrm{O}_{4}$ films," Phys. Rev. B, vol. 53, pp. 9175-9187, 1996.

[3] W. L. Zhou, K.-Y. Wang, C. J. O'Conner, and J. Tang, "Granular growth of $\mathrm{Fe}_{3} \mathrm{O}_{4}$ thin films and its antiphase boundaries prepared by pulsed laser deposition," J. Appl. Phys., vol. 89, pp. 7398-7400, 2001.

[4] Y. Zhou, X. Jin, and I. V. Shvets, "Enhancement of the magnetization saturation in magnetite (100) epitaxial films by thermo-chemical treatment," J. Appl. Phys., vol. 95, pp. 7357-7359, 2004.

[5] — - "Effect of thermo-chemical treatment on magnetic and spin-transport properties of epitaxial $\mathrm{Fe}_{3} \mathrm{O}_{4}$ (100)/MgO films," J. Magn. Magn Mat., vol. 286, pp. 346-350, 2005.

[6] Y. Zhou, X. Jin, Y. M. Mukovskii, and I. V. Shvets, "Kinetics of oxidation of low-index surfaces of magnetite," J. Phys.: Condensed Matter, vol. 16, pp. 1-12, 2004.

[7] A. Koblischka-Veneva, M. R. Koblischka, F. Mücklich, N. Hari Babu, and D. A. Cardwell, "Comparative study of grain orientation in melttextured HTSC with different additions," Physica C, vol. 426-431, pp. 618-624, 2005.

[8] A. Koblischka-Veneva, M. R. Koblischka, F. Mücklich, K. Ogasawara, and $\mathrm{M}$. Murakami, "Interactions of $\mathrm{Y}_{2} \mathrm{BaCuO}_{5}$ particles and the YBCO matrix within melt-textured YBCO samples studied by means of electron backscatter diffraction," Supercond. Sci. Technol., vol. 18, pp. S158-S163, 2005.

[9] Orientation Imaging Microscopy Software Version V4.0, TexSEM Laboratories (TSL), Draper, UT, 2004.

[10] M. R. Koblischka and A. Koblischka-Veneva, "Characterization of bulk superconductors through EBSD methods," Physica C, vol. 392-396, pp. 545-556, 2003.

[11] A. Koblischka-Veneva, M. R. Koblischka, P. Simon, F. Mücklich, K. Ogasawara, and M. Murakami, "Application of electron backscatter diffraction to bulk high- $T_{\mathrm{c}}$ superconductors," Supercond. Sci. Technol., vol. 15 , pp. 796-802, 2002.

[12] T. Tepper, C. A. Ross, and G. F. Dionne, "Microstructure and optical properties of pulsed-laser-deposited iron oxide films," IEEE Trans. Magn., vol. 40, no. 3, pp. 1685-1690, Mar. 2004.

[13] J. D. Wei, J. D. Wei, I. Knittel, Y. Zhou, S. Murphy, F. T. Parker, I. V. Shvets, and U. Hartmann, "Influence of the anti-phase domain distribution on the magnetic structure of magnetite thin films," Appl. Phys. Lett., 2006, to be published.

Manuscript received March 13, 2006 (e-mail: a.koblischka-veneva@mx.unisaarland.de). 